\title{
miR-181b-5p promotes proliferation and inhibits apoptosis of hypertrophic scar fibroblasts through regulating the MEK/ERK/p21 pathway
}

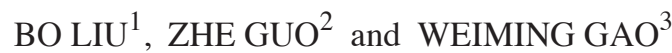 \\ ${ }^{1}$ Department of Medical Cosmetology, Eastern Liaoning University, Dandong, Liaoning 118003; \\ ${ }^{2}$ Department of Dermatology, The First Hospital of China Medical University, Shenyang, Liaoning 110000; \\ ${ }^{3}$ Department of Medicine, Eastern Liaoning University, Dandong, Liaoning 118003, P.R. China
}

Received June 4, 2018; Accepted October 26, 2018

DOI: $10.3892 / \mathrm{etm} .2019 .7159$

\begin{abstract}
Hypertrophic scar (HS) is a common skin disorder occurring during the wound healing process, and the pathogenesis of HS remains unclear. Previous studies indicated that miRNAs may be involved in the onset and progression of HS. In the present study, reverse transcription-quantitative polymerase chain reaction (RT-qPCR) and western blotting were used to investigate the expression of miR-181b-5p and decorin in HS tissues. Direct interaction between miR-181b-5p and decorin was confirmed using a dual-luciferase assay. Human HS fibroblasts (HSFbs) were cultured and transfected with miR-181b-5p mimics, and MTT assay and Annexin V fluorescein isothiocyanate/propidium iodide staining were performed to investigate the role of miR-181b-5p in the proliferation and apoptosis of HSFbs. Subsequently, the expression levels of mitogen-activated protein kinase kinase (MEK), phospho (p)-extracellular signal-regulated kinase (ERK) and p21 were determined in HSFbs transfected with miR-181b-5p mimics and untransfected cells using RT-qPCR and western blotting. The results indicated upregulation of miR-181b-5p and downregulation of decorin expression in HS tissues compared with normal skin samples. miR-181b-5p may regulate the expression of decorin through direct binding to the 3'-untranslated region, as demonstrated by the results of the dual-luciferase assay. Transfection with miR-181b-5p mimics in HSFbs enhanced cell proliferation, reduced apoptosis and increased the expression of MEK, p-ERK and p21. Furthermore, treatment with MEK inhibitor in HSFbs transfected with miR-181b-5p mimics partially inhibited miR-181b-5p-induced antiapoptotic
\end{abstract}

Correspondence to: Professor Bo Liu, Department of Medical Cosmetology, Eastern Liaoning University, 325 Wenhua Road, Dandong, Liaoning 118003, P.R. China

E-mail: niuBo9915@163.com

Key words: microRNA-181b-5p, proliferation, apoptosis, hypertrophic fibroblasts, decorin, mitogen-activated protein kinase kinase/extracellular signal-regulated kinase/p21 pathway effects. Taken together, increased expression of miR-181b-5p may serve important roles in the pathogenesis of HS through regulating the MEK/ERK/p21 pathway, suggesting that miR-181b-5p may be a therapeutic target for the treatment of HS.

\section{Introduction}

Hypertrophic scar (HS) is a common fibroproliferative disorder that may develop following thermal or traumatic injuries involving reticular dermis, characterized by excessive extracellular matrix (ECM) deposition and dysregulated activation of fibroblasts (1). Pathological scars frequently affect a patient's quality of life due to functional and/or aesthetic reasons (2). Although the mechanisms underlying the pathogenesis of HS are not well understood, excessive proliferation and apoptosis resistance of HS fibroblasts (HSFbs) serve roles in HS formation $(3,4)$.

MicroRNAs (miRNAs/miRs) are a group of small non-coding RNAs (18-22 nucleotides in length), which regulate the expression of target mRNAs through binding to their 3'-untranslated region (UTR) (5). Previous studies indicated that miRNAs serve roles in the pathophysiological processes of certain skin disorders $(6,7)$. Therefore, the role of miRNAs in HS has been previously investigated $(8,9)$. A recent study indicated that HS tissues exhibit increased levels of miR-181b-5p compared with normal skin (10). Inhibition of miR-181b-5p reverses transforming growth factor- $\beta 1$ (TGF- $\beta 1$ )-induced myofibroblast differentiation in HS, suggesting that miR-181b-5p may be involved in the pathological process of HS (10). A number of studies demonstrated that miR-181b-5p is associated with proliferation of various types of cells, including vascular smooth muscle cells (11), tumor cells (12-14) and metanephric mesenchymal cells (15). Therefore, the present study aimed to investigate whether miR-181b-5p serves a role in the regulation of proliferation and apoptosis of HSFbs.

A search for genes targeted by miR-181b-5p using TargetScan database has revealed a potential binding site for miR-181b-5p in the 3'-UTR of decorin. A previous study confirmed that miR-181b-5p can directly regulate the expression of decorin 
by binding to the predicted target site (10). Decorin, a member of the small leucine-rich proteoglycan family, is an abundant protein in the ECM, where it inactivates profibrotic TGF- $\beta 1$ and regulates cellular functions involved in wound healing and scar formation $(16,17)$. Decreased decorin expression level was identified in HS tissues compared with normal skin samples (10), and recombinant decorin was demonstrated to induce an in vitro and in vivo anti-fibrotic effect in HS development (18-20). Decorin exerts its beneficial functions partially through inhibiting proliferation and inducing apoptosis of HSFbs $(19,20)$. By targeting decorin, miR-181b-5p may serve a role in proliferation and apoptosis of HSFbs during HS formation.

The present study aimed to confirm the dysregulated expression of miR-181b-5p and its target decorin in HS tissues and the direct interaction between miR-181b-5p and decorin. Furthermore, the effect of miR-181b-5p on proliferation and apoptosis of HSFbs was also determined in the present study. The results of the present study indicated that the mitogen-activated protein kinase kinase (MEK)/extracellular signal-regulated kinase (ERK)/p21 pathway may serve a role in the mechanisms underlying the effect of miR-181b-5p on HSFbs. These results may improve the understanding of the molecular mechanisms of HS and provide novel diagnostic and predictive markers for HS therapy.

\section{Materials and methods}

Tissue samples. A total of 30 pairs of HS tissues and adjacent normal skin tissues were obtained from patients who underwent plastic surgery in the Department of Dermatology, The First Hospital of China Medical University (Shenyang, China), and the diagnosis of HS was confirmed using routine pathological approaches as previously described (21). All experiments were approved by the Ethics Committee of Eastern Liaoning University (Dandong, China), and written informed consent was obtained from all patients.

Cell culture. Human HS fibroblasts (HSFbs; Shanghai Bioleaf Biotech, Co., Ltd., Shanghai, China) were cultured in Dulbecco's modified Eagle's medium (Thermo Fisher Scientific, Inc., Waltham, MA, USA) supplemented with $10 \%$ fetal bovine serum (Thermo Fisher Scientific, Inc.) and 1\% (w/v) penicillin/streptomycin (Sigma-Aldrich; Merck KGaA, Darmstadt, Germany) in a humidified incubator with $5 \% \mathrm{CO}_{2}$ at $37^{\circ} \mathrm{C}$.

Cell transfection. HSFbs were transfected with $50 \mathrm{nmol}$ miR-181b-5p mimics or negative control (NC) miRs (Shanghai GenePharma Co., Ltd., Shanghai, China) using Lipofectamine ${ }^{\circledR} 3000$ (Invitrogen; Thermo Fisher Scientific, Inc.) according to the manufacturer's protocol. Cells were harvested $48 \mathrm{~h}$ following transfection for further analysis. To investigate the association between miR-181-5p and the $\mathrm{MEK} / \mathrm{ERK} / \mathrm{p} 21$ signaling pathway, cells transfected with $50 \mathrm{nmol}$ miR-181b-5p mimics were treated with MEK inhibitor trametinib (Selleck Chemicals, Houston, TX, USA). The sequences of the miR-181b-5p mimics and NC were as follows: miR-181b-5p mimics, 5'-AACAUUCAUUGCUGUCGGUGG GU-3'; miR-181b-5p mimics NC, 5'-UUCUCCGAACGUGUC ACGUTT-3'.
Reverse transcription-quantitative polymerase chain reaction (RT-qPCR). Total RNA was extracted using TRIzol reagent (Thermo Fisher Scientific, Inc.) and reverse transcription was performed using PrimeScript ${ }^{\mathrm{TM}}$ RT reagent kit (Takara Biotechnology Co., Ltd., Dalian, China) at $37^{\circ} \mathrm{C}$ for $15 \mathrm{~min}$ and $85^{\circ} \mathrm{C}$ for $5 \mathrm{sec}$. qPCR was performed using $\mathrm{SYBR}^{\circledR}$ Premix Ex Taq $^{\text {TM }}$ kit (Takara Biotechnology Co., Ltd.) with an ABI 7300 Real-Time PCR System (Applied Biosystems; Thermo Fisher Scientific, Inc.) according to manufacturer's protocol. The following thermocycling conditions were used for the qPCR: Initial denaturation at $95^{\circ} \mathrm{C}$ for $30 \mathrm{sec} ; 40$ cycles of $95^{\circ} \mathrm{C}$ for $5 \mathrm{sec}$ and $60^{\circ} \mathrm{C}$ for $30 \mathrm{sec}$. Primers were synthesized by GenScript (Nanjing, China) and are listed in Table I. Relative expression of $\mathrm{p} 21$ in each sample was normalized to the level of GAPDH using the $2^{-\Delta \Delta C q}$ method (22). The expression of miR-181b-5p was determined using the Hairpin-it ${ }^{\mathrm{TM}}$ miRNAs qPCR Quantitation kit (Shanghai GenePharma Co., Ltd.) according to manufacturer's protocol, and RNA, U6 small nuclear 6, pseudogene (GenScript Corporation, Piscataway, NJ, USA) was used for normalization.

Western blotting. Proteins were extracted from the harvested cells using radioimmunoprecipitation assay (Beyotime Institute of Biotechnology, Haimen, China), and protein concentration was determined using Pierce ${ }^{\circledR}$ BCA Protein Assay kit (Pierce; Thermo Fisher Scientific, Inc.). Proteins were separated using SDS-PAGE (10\% gel), and transferred onto polyvinylidene difluoride membranes. Subsequently, the membranes were blocked at room temperature for $1 \mathrm{~h}$ using 5\% non-fat milk in tris-buffered saline and incubated with primary antibodies: Anti-decorin (cat. no. ab175404), anti-MEK (cat. no. ab178876), anti-ERK (cat. no. ab54230), anti-p-ERK (cat. no. ab222493) and anti-p21 (cat. no. ab109520; all 1:1,000; Abcam, Cambridge, MA, USA) at $4^{\circ} \mathrm{C}$ overnight. The membranes were washed and incubated with secondary antibodies (horseradish peroxidase-conjugated goat anti-rabbit; cat. no. ab6721; 1:5,000; Abcam). Bands were visualized by incubation with Novex ${ }^{\circledR}$ ECL Chemiluminescent Substrate Reagent kit (Thermo Fisher Scientific, Inc.), and the signals were detected using ChemiDoc $^{\text {TM }}$ XRS+ imaging system (Bio-Rad Laboratories, Inc., Hercules, CA, USA).

Cell apoptosis assay. Cell apoptosis was evaluated using Annexin V-fluorescein isothiocyanate (FITC)/propidium iodide (PI) apoptosis detection kit (BD Biosciences, Franklin Lakes, NJ, USA). Cells were washed with ice-cold PBS, resuspended in cold Annexin $\mathrm{V}$ binding buffer, and incubated with Annexin V/FITC and PI for $15 \mathrm{~min}$ at room temperature. The percentage of apoptotic cells was analyzed using BD FACSVerse ${ }^{\mathrm{TM}}$ flow cytometer (BD Biosciences). The data was analyzed by FlowJo (version 7.6.5; Tree Star, Inc., Ashland, OR, USA).

Cell proliferation assay. Cell proliferation was measured using MTT assay (Sigma-Aldrich; Merck KGaA) in a 96-well plate according to the manufacturer's protocol. MTT solution was added to each well and the plate was incubated at $37^{\circ} \mathrm{C}$ for $4 \mathrm{~h}$. The optical density of each well was measured using a microplate reader at a wavelength of $490 \mathrm{~nm}$. 
Table I. Sequences of the primers used in the reverse transcription-quantitative polymerase chain reaction analysis.

Primer sequence $\left(5^{\prime}-3^{\prime}\right)$

\begin{tabular}{lll}
\cline { 2 - 3 } Name & \multicolumn{1}{c}{ Forward } & \multicolumn{1}{c}{ Reverse } \\
\hline miR-181b & GCGGATCATTCATTGCTGTCG & ATCTGGTGGCTCTCGGAGTAA \\
Decorin & CGGAATTCACATGAAGGCCACTATCATCCT & CGGGATCCTTATAGTTTCCGAGTTAATG \\
MEK & GACGACCAGTGGGGAGAGTA & ACAGCGGCTCAACTCTCAAA \\
p21 & ATGTCAGAACCGGCTGGGGA & TTAGGGCTTCCTCTTGGAGA \\
GAPDH & AAGTATGACAACAGCCTCAAGA & CACCACCTTCTTGATGTCATCA \\
U6 & CTCGCTTCGGCAGCACATA & GTGCAGGGTCCGAGGT \\
\hline
\end{tabular}
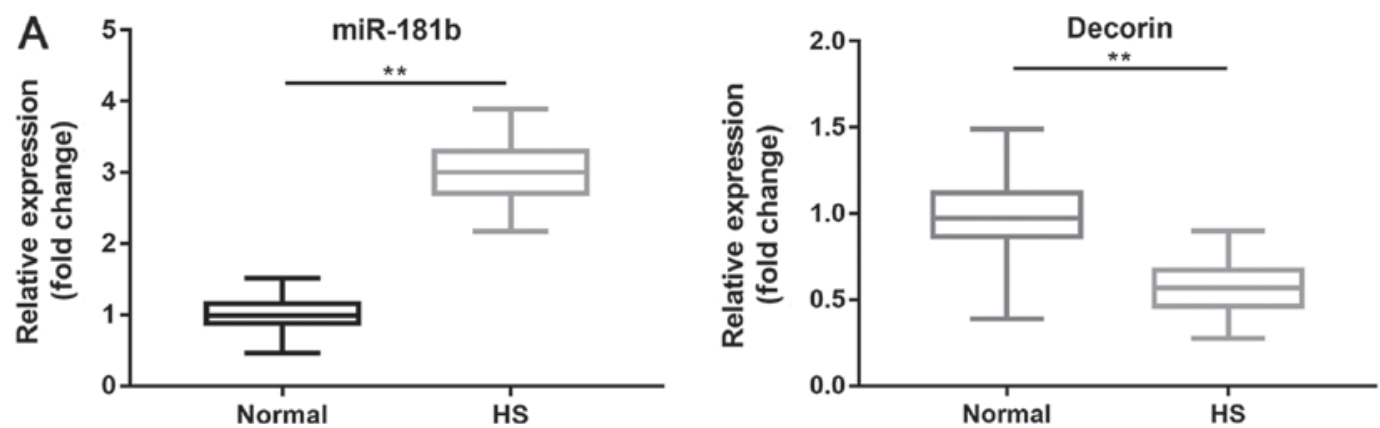

B

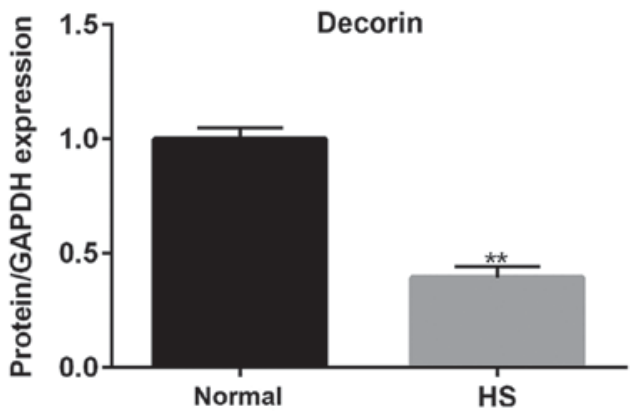

Figure 1. miR-181b-5p is upregulated and negatively associated with decorin expression in HS tissues. (A) Reverse transcription-quantitative polymerase chain reaction analysis of miR-181b-5p and decorin mRNA expression in HS tissues and normal control samples. (B) Representative western blot analysis of the protein expression of decorin. GAPDH was used as the loading control. Data were normalized to the expression level of GAPDH. Results are presented relative to the value of normal control samples. ${ }^{* *} \mathrm{P}<0.01$ vs. the normal control samples. HS, hypertrophic scar.

Prediction of target gene of miR-181b-5p. To predict the target gene of miR-181b-5p, the authors used the online website TargetScan (www.targetscan.org). miR-181b-5p has a binding site at the 3'-UTR of decorin.

Dual luciferase activity assay. The 3'-UTR of decorin with the predicted miR-181b-5p binding site was amplified by PCR from genomic DNA, and inserted into the pMIR-REPORT ${ }^{\mathrm{TM}}$ luciferase reporter vector (Thermo Fisher Scientific, Inc.) to obtain the wild-type luciferase reporter plasmid (p-decorin-wt). Several nucleotides were mutated in the predicted binding region of miR-181b-5p using PCR, which used the Easy-Load ${ }^{\mathrm{TM}}$ PCR Master Mix (Beyotime Institute of Biotechnology); the thermo profile was as follows: Initial denaturation at $94^{\circ} \mathrm{C}$ for $3 \mathrm{~min}, 30$ cycles of denaturation at $94^{\circ} \mathrm{C}$ for $30 \mathrm{sec}$, annealing at $55^{\circ} \mathrm{C}$ for $30 \mathrm{sec}$ and elongation at $72^{\circ} \mathrm{C}$ for $1 \mathrm{~min}$, and final elongation at $72^{\circ} \mathrm{C}$ for $1 \mathrm{~min}$. This fragment was cloned into the pMIR-REPORT luciferase reporter vector to generate a mutant reporter plasmid (p-decorin-mut). All constructed plasmids were verified through DNA sequencing. 293T cells were cultured in 96-well plates and co-transfected with miR-181b-5p or NC, and luciferase reporter plasmid using Lipofectamine 3000. After $24 \mathrm{~h}$, the cells were lysed and their luciferase activities were assayed using Dual-Luciferase ${ }^{\circledR}$ Reporter Assay System (Promega Corporation, Madison, WI, USA). Values were normalized to Renilla luciferase activity.

Statistical analysis. All experiments were performed in triplicate. Data were analyzed using SPSS 20.0 software (IBM Corp., Armonk, NY, USA) and presented as the mean \pm standard deviation. Statistical significance between two groups was analyzed using Student's t-test, and the comparisons among multiple groups were performed using analysis of variance followed by Turkey's post hoc test. $\mathrm{P}<0.05$ was considered to indicate a statistically significant difference. 

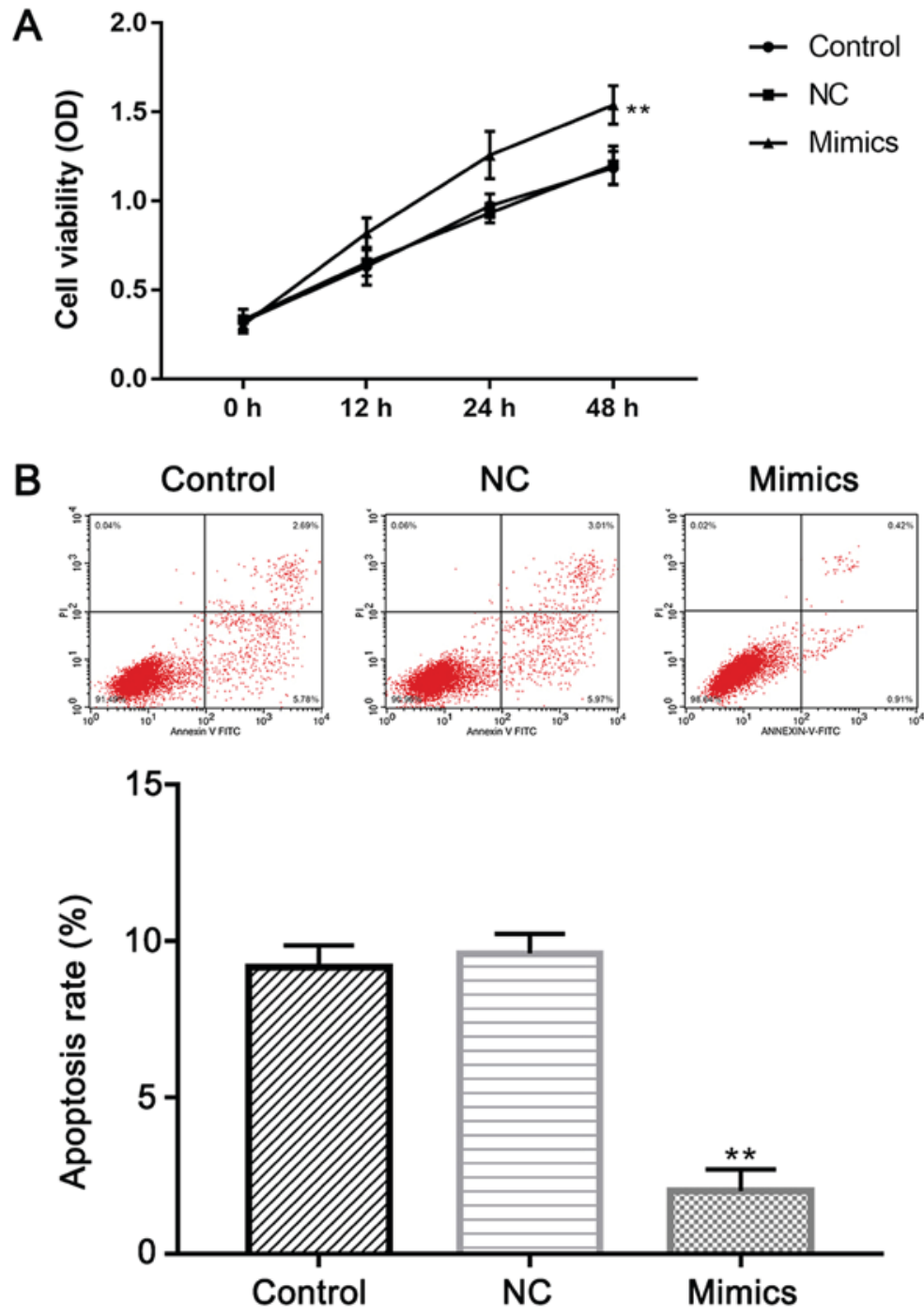

Figure 2. miR-181b-5p increases the proliferation and inhibits apoptosis of human HSFbs. Primary human hypertrophic scar fibroblasts were transfected with miR-181-5p mimics or NC miRs. Untransfected cells were used as the normal control. (A) MTT assay was used to examine cell proliferation with OD values measured at a wavelength of $490 \mathrm{~nm}$. Data are presented as the mean \pm standard deviation of three independent experiments. (B) Representative scatter plots of cell apoptosis assessed using flow cytometry following Annexin V-FITC/PI staining. Apoptotic rates were calculated and are presented as the mean \pm standard deviation from three independent experiments. ${ }^{* *} \mathrm{P}<0.01$ vs. the control group. HSFbs, hypertrophic scar fibroblasts; NC, negative control; miR, microRNA; OD, optical density; FITC, fluorescein isothiocyanate; PI, propidium iodide.

\section{Results}

miR-181b-5p is overexpressed and negatively associated with decorin expression in human HS tissues. RT-qPCR was used to investigate the expression of miR-181b-5p. As indicated in Fig. 1A, miR-181b-5p was present in normal tissue samples; however, its expression increased 2-fold in HS tissues $(\mathrm{P}<0.01)$. Decorin was identified as a candidate target gene of miR-181b-5p based on the results of bioinformatics analysis. RT-qPCR and western blot analysis of decorin expression were performed using the tissue samples. HS tissues exhibited a significantly reduced decorin expression, both at the mRNA and protein level $(\mathrm{P}<0.01$; Fig. $1 \mathrm{~A}$ and $\mathrm{B})$. These data suggested an inverse association between the expression of miR-181b-5p and its target decorin, and are consistent with a previous study (10).

miR-181b-5p induces proliferation and inhibits apoptosis of HSFbs. Increased proliferation and reduced apoptosis contribute to the development of HS (9). To clarify the biological roles of miR-181b-5p in HSFbs, MTT assay and Annexin V-FITC/PI staining were preformed following transfection with miR-181b-5p in HSFbs (Fig. 2). Transfection with miR-181b-5p mimics resulted in a 3 -fold increase in miR-181b-5p miRNA expression (Fig. 3A). MTT assay revealed that miR-181b-5p mimics promoted cell proliferation in a time-dependent manner (Fig. 2A). miR-181b-5p mimics increased cell proliferation at 12 and $24 \mathrm{~h}$ post-transfection compared with untreated control cells or NC miRs. A significant increase in cell proliferation was observed at $48 \mathrm{~h}$ post-transfection $(\mathrm{P}<0.01)$. Flow cytometry analysis using Annexin V-FITC/PI staining (Fig. 2B) indicated that miR-181b-5p significantly inhibited cell apoptosis compared with the control and $\mathrm{NC}$ miR groups $(\mathrm{P}<0.01)$. The percentage of apoptotic HSFbs cells transfected with miR-181b-5p mimics decreased $\sim 4$-fold compared with the NC miR group.

miR-181b-5p may downregulate the expression of decorin via the MEK/ERK/p21 pathway. As demonstrated above, 

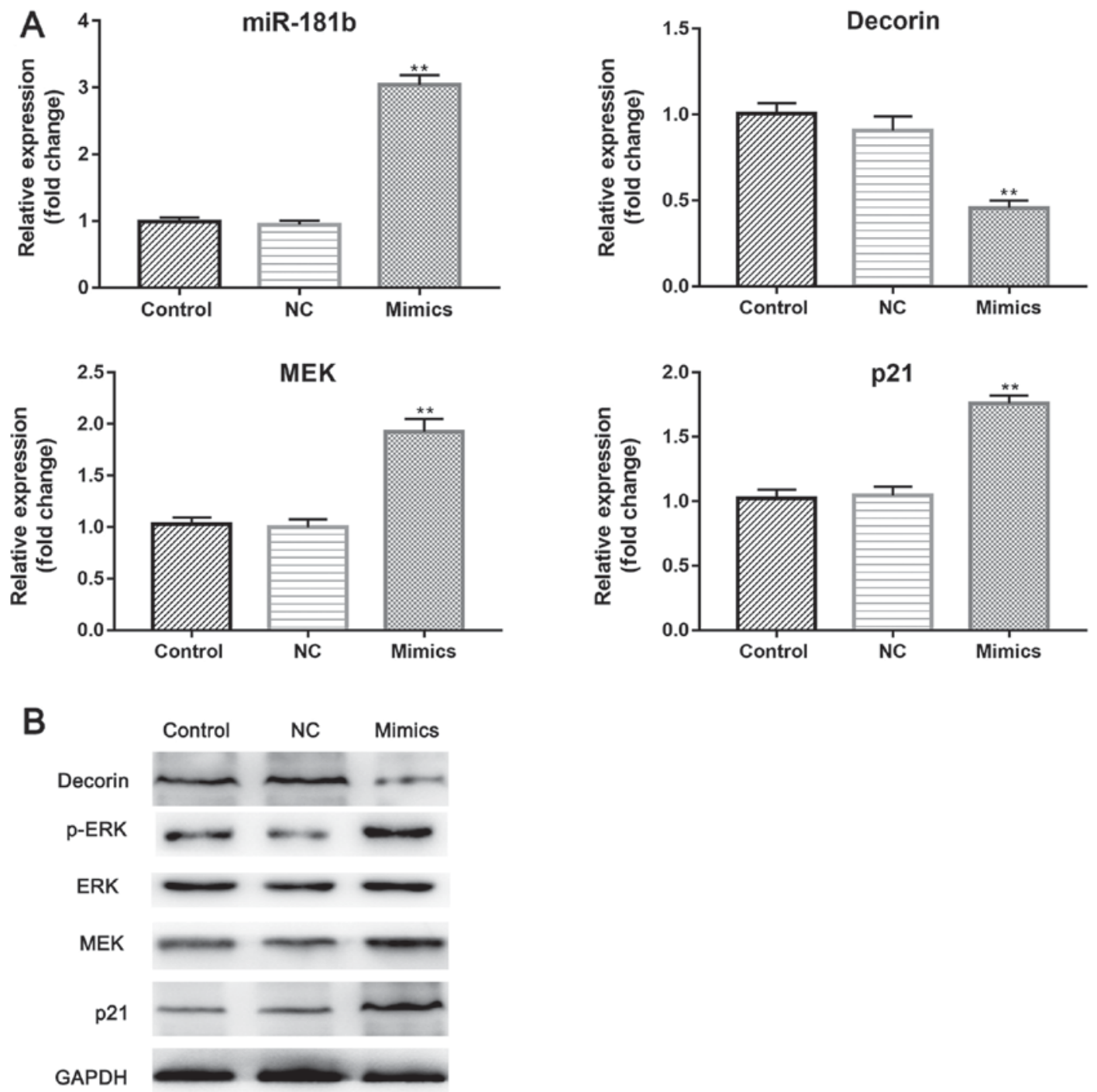

Figure 3. miR-181b-5p downregulates the expression of decorin via the ERK/MEK/p21 pathway. (A) Reverse transcription-quantitative polymerase chain reaction analysis of miR-181b-5p, decorin, MEK and p21 mRNA expression in human HSFbs transfected with miR-181b-5p mimic or NC miRs and untransfected cells as the normal control. Data were normalized with the level of GAPDH control. Results were expressed relative to the value of normal controls that were assigned a value of $1 .{ }^{* *} \mathrm{P}<0.01$ vs. the control group. (B) A representative western blot presenting the protein expression of decorin, p-ERK, ERK, MEK and p21 and in human HSFbs transfected with miR-181b-5p mimic or NC miRs, and untransfected cells as the normal control. GAPDH was used as the loading control. HSFbs, hypertrophic scar fibroblasts; NC, negative control; miR, microRNA; MEK, mitogen-activated protein kinase kinase; ERK, extracellular signal-regulated kinase.

miR-181-5p expression was negatively associated with decorin expression. Therefore, the present study aimed to further determine whether the expression of decorin was suppressed following transfection with miR-181b-5p mimics. Transfection with miR-181b-5p mimics induced a significant decrease in decorin expression compared with the NC miR group, both at the mRNA and protein level $(\mathrm{P}<0.01$; Fig. 3$)$.

The present study further aimed to analyze the possible mechanisms underlying miR-181b-5p-mediated cellular responses. The MEK/ERK signaling pathway has been reported to upregulate the expression of p21 (23) and subsequent cellular processes including cell proliferation and apoptosis. It was reported that the activation of MEK/ERK signaling pathway increased the proliferation and decreased the apoptosis of HSFbs (9). To investigate the contribution of miR-181b-5p to the regulation of the MEK/ERK/p21 signaling pathway, the present study determined the expression of MEK,
ERK, p-ERK and p21 in cells transfected with miR-181b-5p using RT-qPCR and western blotting. miR-181b-5p mimics induced a significant increase in mRNA expression of MEK and p21 compared with the NC group $(\mathrm{P}<0.01$; Fig. 3$)$, and similar effects were observed for protein expression of MEK, p-ERK and p21. miR-181b-5p had no effect of the expression of total ERK. Furthermore, cells transfected with miR-181b-5p were treated with MEK inhibitor (Fig. 4). MEK inhibitor partially inhibited the antiapoptotic effects of miR-181b-5p. These results suggest that miR-181b-5p may exert its cellular functions partially through the activation of the MEK/ERK/p21 pathway.

Decorin is a direct target of miR-181b-5p. The present study aimed to confirm whether the observed reduction in decorin mRNA and protein expression resulted from a direct interaction between miR-181b-5p and 3'-UTR of decorin. 

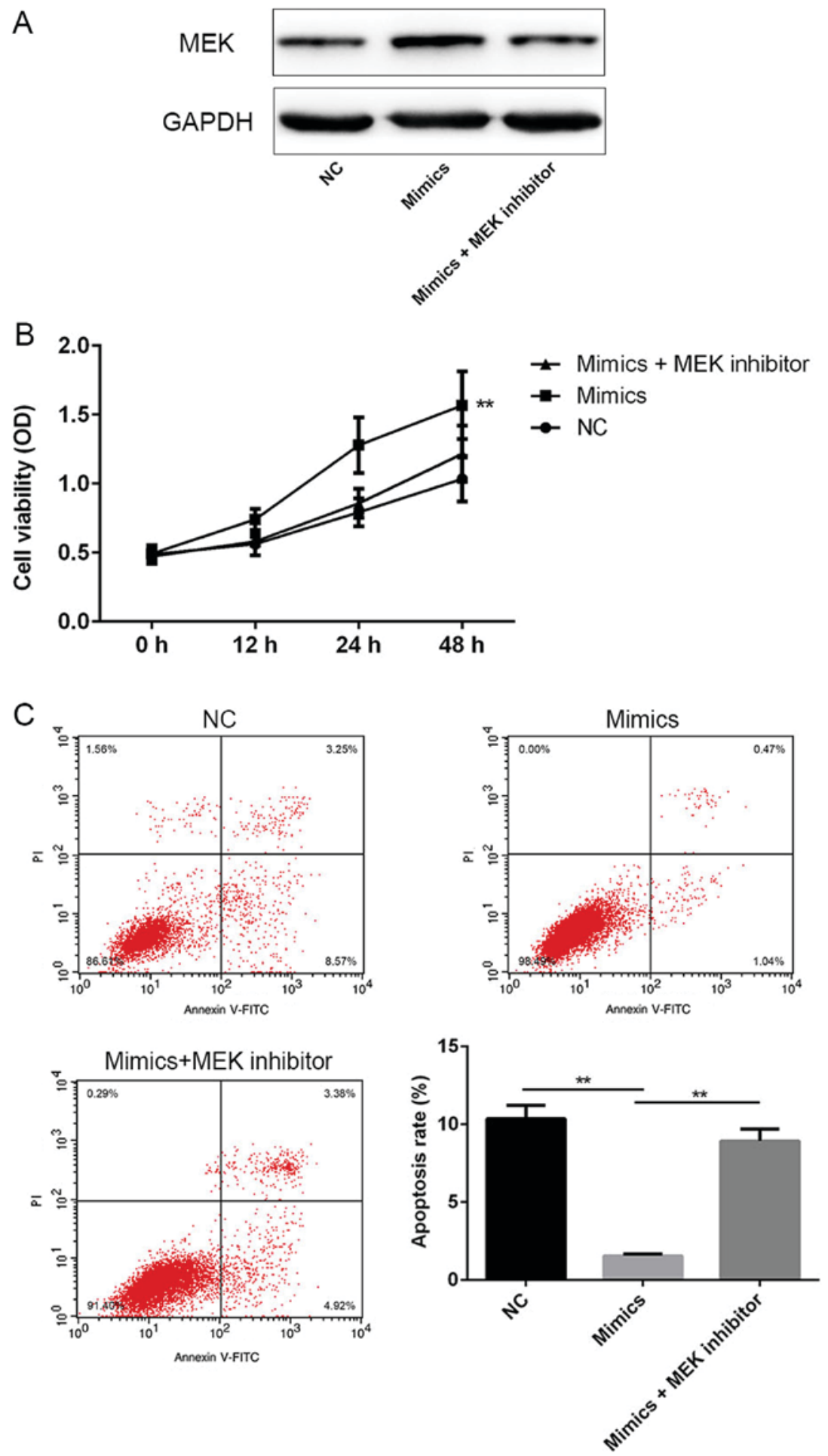

Figure 4. MEK inhibitor partially inhibits miR-181b-5p-induced antiapoptotic effects. (A) Effect of MEK inhibitor on the expression of MEK in cells transfected with miR-181b-5p. (B) Effect of MEK inhibitor on the proliferation of cells transfected with miR-181b-5p. ${ }^{* *} \mathrm{P}<0.01 \mathrm{vs}$. the NC group. (C) Effect of MEK inhibitor on the apoptosis of cells transfected with miR-181b-5p. ${ }^{* *} \mathrm{P}<0.01$ as indicated. Data are presented as the mean \pm standard deviation of three independent experiments. MEK, mitogen-activated protein kinase kinase; NC, negative control; miR, microRNA; OD, optical density; FITC, fluorescein isothiocyanate; PI, propidium iodide.

P-decorin-wt and p-decorin-mut were co-transfected with either miR-181b-5p mimic or NC into $293 \mathrm{~T}$ cells and luciferase activity was measured $24 \mathrm{~h}$ following transfection. Co-transfection with miR-181b-5p mimics and p-decorin-wt significantly decreased the relative luciferase activity compared with the group transfected with NC siR (Fig. 5), whereas a reversal of relative luciferase activity was observed in experiments where miR-181b-5p was co-transfected with reporter construct p-decorin-mut (Fig. 5). The above results indicate that miR-181b-5p could specifically bind to the 3'-UTR of decorin and these results are consistent with a previous study (10). 


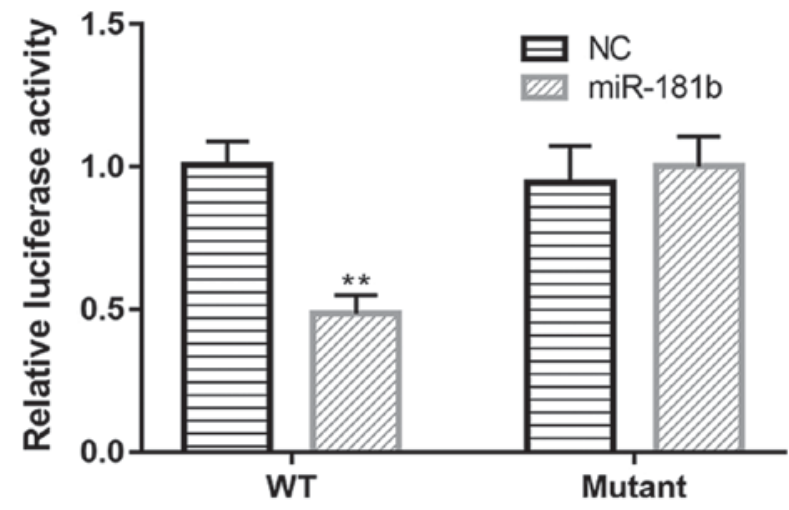

Figure 5. Decorin is a direct target of miR-181b-5p. Each reporter construct (WT or mutant) was co-transfected with either miR-181b-5p mimic or NC miR in $293 \mathrm{~T}$ cells and a dual luciferase assay was performed after $24 \mathrm{~h}$. Luciferase activity was normalized to Renilla and presented as relative to miR NC (assigned a value of 1). Data are presented as the mean \pm standard deviation of three independent experiments. ${ }^{* *} \mathrm{P}<0.01$ vs. the $\mathrm{NC}$ group. miR, microRNA; NC, negative control; WT, wild type.

\section{Discussion}

Fibroblasts serve roles in tissue repair and wound healing processes. Enhanced proliferation and reduced apoptosis of HSFbs have been reported in the development of HS $(3,4)$. Elucidation of the molecular mechanisms underlying proliferation and apoptosis of HSFbs may aid in the development of novel therapeutic strategies that minimize fibrosis and reduce HS formation.

Recently, miRNAs have been demonstrated to regulate various pathological processes in HS $(8,9)$. miR-181b-5p and its target decorin have been hypothesized to be involved in the pathological process of HS (10,18-20). The expression of decorin was markedly downregulated and the mRNA levels of miR-181b-5p were upregulated in HS tissues compared with normal control samples used in the present study. Furthermore, mRNA and protein expression of decorin decreased in HSFbs transfected with miR-181b-5p mimics. The direct interaction between miR-181b-5p and decorin was confirmed using a dual-luciferase assay. The results of the present study confirm previously reported data (10) and suggest that miR-181b-5p may downregulate the expression of decorin via direct binding to the 3'-UTR, contributing to the development of HS.

Considering the proapoptotic and antiproliferative effects of decorin on HSFbs $(19,20)$, the present study hypothesized that miR-181b-5p may exhibit opposite cellular functions. MTT and Annexin V-FITC/PI staining assays were performed to confirm this hypothesis. Significantly enhanced apoptosis and reduced proliferation were observed following transfection with miR-181b-5p mimics in HSFbs. The results of the present study and previously published data (10) indicate that miRNA-181b-5p may be involved in the pathogenesis of HS by promoting fibroblast-to-myofibroblast differentiation, and promoting proliferation and inhibiting apoptosis of fibroblasts.

The intracellular signaling pathway MEK/ERK regulates a number of cellular functions including differentiation, proliferation and apoptosis in numerous types of cells $(24,25)$. Activation of the MEK/ERK pathway leads to the accumulation of p53 and p21, which promotes proliferation and inhibits apoptosis $(9,24)$. Previous studies indicated that the $\mathrm{MEK} / \mathrm{ERK} / \mathrm{p} 21$ pathway is involved in the proliferation and apoptosis of fibroblasts and is associated with the pathogenesis of HS (9,26). Furthermore, the association between miR-181b-5p and the MEK (25) and ERK (26) signaling pathways has been previously studied. Therefore, the present study aimed to investigate the potential effect of miR-181b-5p on the MEK/ERK/p21 pathway in HS. The results of RT-qPCR and western blot analysis demonstrated that transfection with miR-181b-5p mimics activated the expression of MEK, and p21 at mRNA and protein levels. Furthermore, treatment with MEK inhibitor partially inhibited the antiapoptotic effects of miR-181b-5p. These results suggested that miR-181b-5p may exert its function in HS via the MEK/ERK/p21 pathway.

The present study confirmed the increased expression of miR-181b-5p in HS tissues and the direct interaction between miR-181b-5p and decorin, as previously reported $(10,18)$. Furthermore, in the present study, miR-181b-5p mimics significantly inhibited apoptosis, enhanced proliferation of $\mathrm{HSFb}$ and activated the MEK/ERK/p21 signaling pathway. The results of the present study suggest a novel mechanism underlying HS, and miR-181b-5p may be a therapeutic target for prevention and treatment of this disease.

\section{Acknowledgements}

Not applicable.

\section{Funding}

The present study was funded by the Scientific Research Fund of Eastern Liaoning University (grant no. 2017FW022).

\section{Availability of data and materials}

The datasets used and/or analyzed during the present study are available from the corresponding author on reasonable request.

\section{Authors' contributions}

BL performed the experiments and wrote part of the manuscript. ZG performed some of the experiments. WG designed the study and wrote most of the experiments. All authors read and approved the manuscript and agree to be accountable for all aspects of the research in ensuring that the accuracy or integrity of any part of the work are appropriately investigated and resolved.

\section{Ethics approval and consent to participate}

The present study was approved by the ethics committee of Eastern Liaoning University and written informed consent was obtained from all patients.

\section{Patient consent for publication}

The consent for publication was obtained from the patients.

\section{Competing interests}

The authors declare that they have no competing interests. 


\section{References}

1. Gras C, Ratuszny D, Hadamitzky C, Zhang H, Blasczyk R and Figueiredo C: miR-145 contributes to hypertrophic scarring of the skin by inducing myofibroblast activity. Mol Med 21: 296-304, 2015

2. Brown BC, McKenna SP, Siddhi K, McGrouther DA and Bayat A: The hidden cost of skin scars: Quality of life after skin scarring. J Plast Reconstr Aesthet Surg 61: 1049-1058, 2008.

3. Aarabi S, Longaker MT and Gurtner GC: Hypertrophic scar formation following burns and trauma: New approaches to treatment. PLoS Med 4: e234, 2007.

4. Wang XQ, Song F and Liu YK: Hypertrophic scar regression is linked to the occurrence of endothelial dysfunction. PLoS One 12: e0176681, 2017.

5. Bartel DP: MicroRNAs: Genomics, biogenesis, mechanism, and function. Cell 116: 281-297, 2004.

6. Fiedler J, Grönniger E, Pfanne A, Brönneke S, Schmidt K, Falk CS, Wenck H, Terstegen L, Thum T and Winnefeld M: Identification of miR-126 as a new regulator of skin ageing. Exp Dermatol 26: 284-286, 2017.

7. Zhang L, Ge Y and Fuchs E: miR-125b can enhance skin tumor initiation and promote malignant progression by repressing differentiation and prolonging cell survival. Genes Dev 28 2532-2546, 2014.

8. Bi S, Chai L, Yuan X, Cao C and Li S: MicroRNA-98 inhibits the cell proliferation of human hypertrophic scar fibroblasts via targeting Col1A1. Biol Res 50: 22, 2017.

9. Dong $\mathrm{S}$ and Sun Y: MicroRNA-22 may promote apoptosis and inhibit the proliferation of hypertrophic scar fibroblasts by regulating the mitogen-activated protein kinase kinase/extracellular signal-regulated kinase/p21 pathway. Exp Ther Med 14: 3841-3845, 2017.

10. Kwan P, Ding J and Tredget EE: MicroRNA 181b regulates decorin production by dermal fibroblasts and may be a potential therapy for hypertrophic scar. PLoS One 10: e0123054, 2015.

11. Li TJ, Chen YL, Gua CJ, Xue SJ, Ma SM and Li XD: MicroRNA $181 \mathrm{~b}$ promotes vascular smooth muscle cells proliferation through activation of PI3K and MAPK pathways. Int J Clin Exp Pathol 8: 10375-10384, 2015.

12. Shi ZM, Wang XF, Qian X, Tao T, Wang L, Chen QD, Wang XR, Cao L, Wang YY, Zhang JX, et al: MiRNA-181b suppresses IGF-1R and functions as a tumor suppressor gene in gliomas. RNA 19: 552-560, 2013

13. He L, Yao H, Fan LH, Liu L, Qiu S, Li X, Gao JP and Hao CQ: MicroRNA-181b expression in prostate cancer tissues and its influence on the biological behavior of the prostate cancer cell line PC-3. Genet Mol Res 12: 1012-1021, 2013.

14. Zhi F, Wang Q, Deng D, Shao N, Wang R, Xue L, Wang S, Xia X and Yang Y: MiR-181b-5p downregulates NOVA1 to suppress proliferation, migration and invasion and promote apoptosis in astrocytoma. PLoS One 9: e109124, 2014.
15. lyu Z, Mao Z, Wang H, Fang Y, Chen T, Wan Q, Wang M, Wang N, Xiao J, Wei H, et al: MiR-181b targets Six2 and inhibits the proliferation of metanephric mesenchymal cells in vitro. Biochem Biophys Res Commun 440: 495-501, 2013.

16. Honardoust D, Varkey M, Hori K, Ding J, Shankowsky HA and Tredget EE: Small leucine-rich proteoglycans, decorin and fibromodulin, are reduced in postburn hypertrophic scar. Wound Repair Regen 19: 368-378, 2011.

17. Kresse $\mathrm{H}$ and Schönherr E: Proteoglycans of the extracellular matrix and growth control. J Cell Physiol 189: 266-274, 2001

18. Wang P, Liu X, Xu P, Lu J, Wang R and Mu W: Decorin reduces hypertrophic scarring through inhibition of the TGF- $\beta 1 / \mathrm{Smad}$ signaling pathway in a rat osteomyelitis model. Exp Ther Med 12: 2102-2108, 2016

19. Honardoust D, Ding J, Varkey M, Shankowsky HA and Tredget EE: Deep dermal fibroblasts refractory to migration and decorin-induced apoptosis contribute to hypertrophic scarring. J Burn Care Res 33: 668-677, 2012.

20. Zhang Z,Li XJ, Liu Y,Zhang X, Li YY and Xu WS: Recombinant human decorin inhibits cell proliferation and downregulates TGF-betal production in hypertrophic scar fibroblasts. Burns 33: 634-641, 2007.

21. Shi J, Li J, Guan H, Cai W, Bai X, Fang X, Hu X, Wang Y, Wang $\mathrm{H}$, Zheng Z, et al: Anti-fibrotic actions of interleukin-10 against hypertrophic scarring by activation of PI3K/AKT and STAT3 signaling pathways in scar-forming fibroblasts. PLoS One 9: e98228, 2014.

22. Livak KJ and Schmittgen TD: Analysis of relative gene expression data using real-time quantitative PCR and the $2^{-\Delta \Delta C \mathrm{~T}}$ method. Methods 25: 402-408, 2001.

23. Karkhanis M and Park JI: Sp1 regulates Raf/MEK/ERK-induced p21 $1^{\text {CIP1 }}$ transcription in TP53-mutated cancer cells. Cell Signal 27: 479-486, 2015

24. Lin Z, Zhang C, Zhang M, Xu D, Fang Y, Zhou Z, Chen X, Qin $N$ and Zhang $X$ : Targeting cadherin-17 inactivates Ras/Raf/MEK/ERK signaling and inhibits cell proliferation in gastric cancer. PLoS One 9: e85296, 2014.

25. Ramos JW: The regulation of extracellular signal-regulated kinase (ERK) in mammalian cells. Int J Biochem Cell Biol 40: 2707-2719, 2008

26. Li Y,Kilani RT, Rahmani-Neishaboor E, Jalili RB and Ghahary A: Kynurenine increases matrix metalloproteinase- 1 and -3 expression in cultured dermal fibroblasts and improves scarring in vivo. J Invest Dermatol 134: 643-650, 2014. 